\title{
6996
}

\section{A MODIFIED MONTE CARLO MODEL FOR THE IONOSPHERIC HEATING RATES}

\author{
H. G. MAYR \\ E. G. FONTHEIM \\ S. C. ROBERTSON
}

(NASA-TH-X-65980) A MODIFIED MONTE CARLO

MODEL FOR THE IONOSPHERIC HEATING RATES

H.G. Mayr $r_{\theta}$ et al (NASA) Jul. $197212 \mathrm{p}$

\section{JULY 1972}




\title{
A MODIFIED MONTE CARLO MODEL FOR THE IONOSPHERIC HEATING RATES
}

\author{
H. G. Mayr and E. G. Fontheim* \\ Laboratory for Planetary Atmospheres \\ NASA, Goddard Space Flight Center \\ Greenbelt, Md.
}

and

S. C. Robertson

Consultants and Designers, Inc.

Greenbelt, Md.

July 1972

*NAS-NRC Senior Research Associate

GODDARD SPACE FLIGHT CENTER

Greenbelt, Maryland 


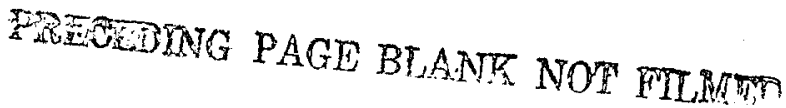

\author{
A MODIFIED MONTE CARLO MODEL FOR THE \\ IONOSPHERIC HEATING RATES
}

\begin{abstract}
Following Cicerone and Bowhill (1970a, b, 1971) a Monte Carlo method is adopted as a basis for the derivation of the photoelectron heat input into the ionospheric plasma. Considering that the size of the source elements must be small compared with the range over which photoelectrons dissipate their energy, a great number of Monte Carlo runs are required normally for the computation of the heating rates. This approach is modified in an attempt to minimize the computation time. The heat input distributions are computed for arbitrarily small source elements that are spaced at distances apart corresponding to the photoelectron dissipation range. By means of a nonlinear interpolation procedure their individual heating rate distributions are utilized to produce synthetic ones that fill the gaps between the Monte Carlo generated distributions. By varying these gaps and the corresponding number of Monte Carlo runs the accuracy of the results is tested to verify the validity of this procedure. It is concluded that this model can reduce the computation time by more than a factor of three, thus improving the feasibility of including Monte Carlo calculations in self-consistent ionosphere models.
\end{abstract}




\section{A MODIFIED MONTE CARLO MODEL FOR THE IONOSPHERIC HEATING RATES}

Recently Mayr et al. (1972) have developed an iterative method to solve the ionospheric equations for $\mathrm{H}^{+}, \mathrm{O}^{+}, \mathrm{He}^{+}$, and electrons. In this approach an assumed temperature profile is substituted into the continuity and momentum equations in order to calculate the composition and velocity profiles. The energy equation is then solved for the temperature by using the calculated densities and velocities. In the second iteration, the temperature profile from the first iteration is substituted into the continuity and momentum equations resulting in improved composition and velocity profiles etc. Since the electron heat input profile is a function of the electron density, the heat input profile has to be recalculated in each iteration if the calculation is to be self-consistent.

Our estimates have shown that one would require about $3-4$ iterations to achieve convergence on a solution which is accurate to less than $10 \%$ and this implies that the heat input calculations have to be performed several times just to get one self-consistent solution.

The problem is therefore to adopt a heat input model which is both realistic and reasonably efficient. To provide such a model, we have chosen a Monte Carlo approach which was introduced into photoelectron calculations by Cicerone and Bowhill (1970a, b; 1971). The advantages of this method are obvious: there 
are essentially no limitations on the physical processes than can be considered, including elastic and inelastic collisions, angular scattering and so on.

In the sample calculations presented here, the photoelectron production spectrum of Figure 1 has been used, and the altitide profile has been obtained from this spectrum by scaling it with the scale height of atomic oxygen.

Figure 2 shows four heat input histograms due to photoelectrons that are released at discrete altitudes indicated by the dashed lines. The calculations are based on an ambient electron density of $2 \times 10^{6} \mathrm{~cm}^{-3}$ at $200 \mathrm{~km}$, falling off with a scale height of $150 \mathrm{~km}$. The electron impact cross sections have been obtained from Cicerone and Bowhill (1970a). The neutral model atmosphere of Jacchia (1964) with $\mathrm{T}_{\infty}=800^{\circ} \mathrm{K}$ has been used。

Figure 2 clearly shows the dominance of local heating at lower altitudes where the range of the energy dissipation is smali compared with the scale height. At higher altitudes the histograms widen, thus indicating the importance of non-local heating. The resuits shown in Figure 2 also include the effect of conjugate heating under the assumption of symmetry about the equatorial plane.

In performing these Monte Carlo calculations one must require that the height interval or the volume element out of which photoelectrons are released is small compared with the dissipation range or the width of the histogram. At higher altitudes where non-local heating dominates, this height element could be 
perhaps $20 \mathrm{~km}$. But at lower altitudes, even in the transition region between local and non-local heating, this height element shrinks to less than a kilometer. This constitutes a serious drawback if one relies entirely on a Monte Carlo model to calculate the complete heat input profile because a large number of histograms would have to be generated at those altitudes where the dissipation range is small. This, of course, would be a rather time-consuming process.

For this reason the heat-input calculations have been modified. The modification is based on the hypothesis that the shape of these histograms varies in a continuous fashion with altitude. The peak value decreases with increasing altitude, corresponding to the decrease in the neutral and ionospheric densities, and the width of the histograms broadens toward higher altitudes.

By assuming continuity between histograms, the entire heat input profile can be deduced by means of an interpolation scheme and the information content of only a few Monte Carlo histograms. Therefore, our procedure is to generate a few histograms at discrete altitudes and then to construct the histograms at all remaining altitudes with a non-linear interpolating algorithm. The heat input profile for the entire ionosphere is then simply the sum of the contributions of both the Monte Carlo and interpolated histograms.

The computation time to generate one interpolated histogram is a fraction of the time needed for an actual Monte Carlo generated histogram. 
To demonstrate the validity of this scheme Figure 3 shows two histograms. The one in solid lines was generated by the Monte Carlo method at $200 \mathrm{~km}$. The one with dashed lines is a synthetic histogram generated through interpolation from the adjacent Monte Carlo generated histograms at 180 and $230 \mathrm{~km}$. Both are in reasonably good agreement except near the tails where the contribution to the total heat input is insignificant. In this case conjugate heating was negligible but in examples from higher altitudes, where conjugate heating becomes important, the agreement is similarly good.

In Figure 4 two heat input distributions are presented. The solid line is based on 8 Monte Carlo histograms at the discrete heights indicated by the lines on the altitude axis. The dashed heat input profile has been generated from 4 Monte Carlo histograms at heights indicated by the dashed lines on the altitude axis. The agreement is very good except perhaps at higher altitudes which is not too surprising considering that in the 4 histogram solution no direct contribution came from $400 \mathrm{~km}$, a height which strongly contributes to non-lor:al heating.

In conclusion it can be said that this scheme seems well justified and that in the framework of an interpolation procedure, the Monte Carlo method can be used efficiently in computing the heat input distribution in the ionosphere.

\section{ACKNOWLEDGMENT}

The photoelectron production program was kindly provided by R. J. Stolarski. 


\section{REFERENCES}

Mayr, H. G., E. G. Fontheim, L. H. Brace, H. C. Brinton, and H. A. Taylor, Jr., "A Theorretical Model of the Ionosphere Dynamics with Interhemispheric Coupling", J. Atm. Terr. Phys. 1972, in press.

Cicerone, R. J. and S. A. Bowhill, "Monte Carlo and Thomson Scatter Plasma Line Studies of Ionospheric Photoelectrons", Aeronomy Report No. 39, Aeronomy Laboratory, Department of Electrical Engineering, University of Illinois, 1970a.

Cicerone, R. J. and S. A. Bowhill, "Photoelectron Escape Fluxes Obtained by a Monte Carlo Technique", Radio Sci. 5, 49, $1970 \mathrm{~b}$.

Cicerone, R. J. and S. A. Bowhill, "Photoelectron Fluxes in the Ionosphere Computed by a Monte Carlo Method", J. Geophys. Res. 76, 8299, 1971. Jacchia, L. G., "Static Diffusion Models of the Upper Atmosphere with Empirical Temperature Profiles", Smithsonian Inst. Astrophys. Obs., Special Report No. 170, 1964. 


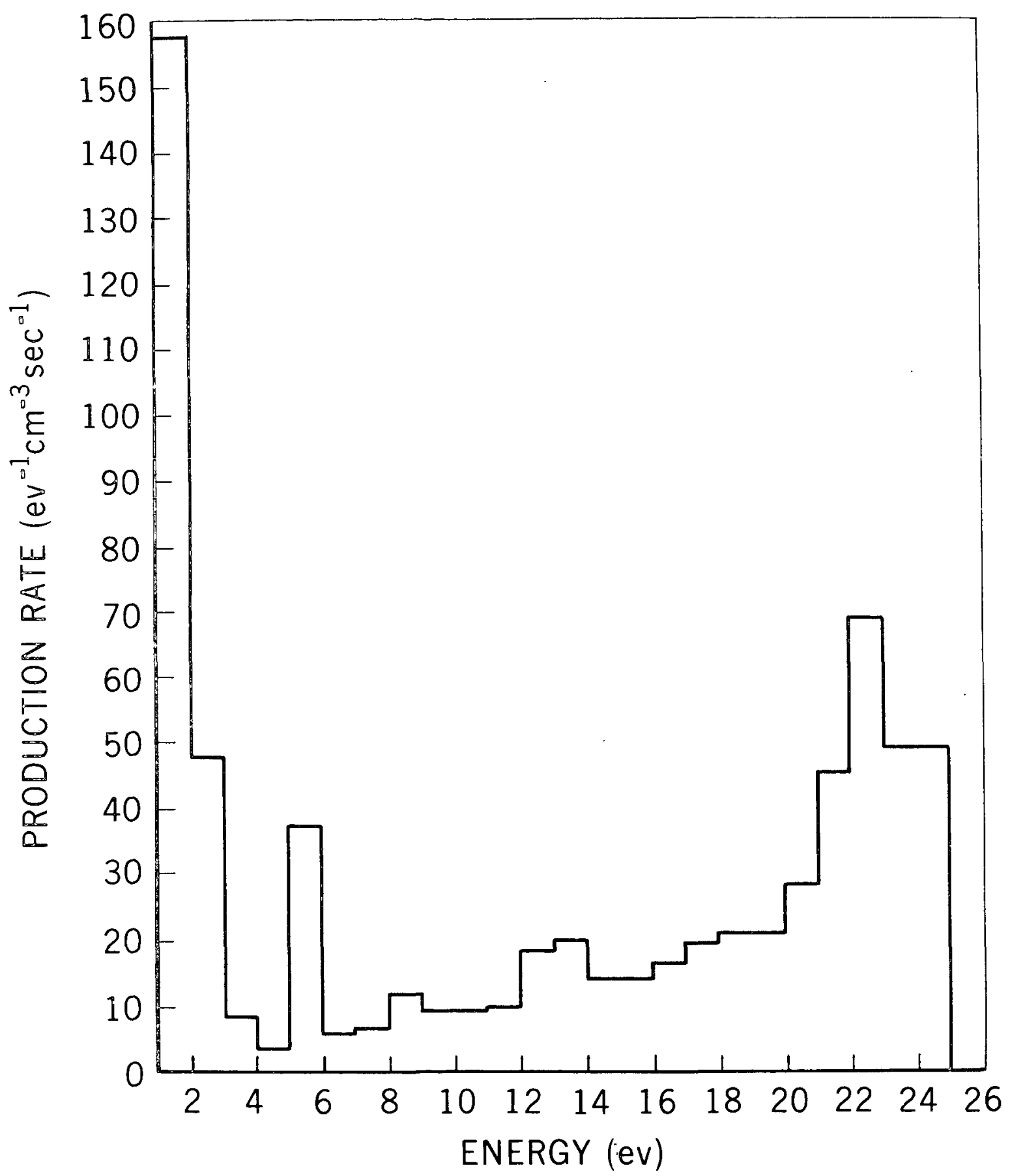

Figure 1. Photoelectron Production Spectrum at $200 \mathrm{~km}$. 


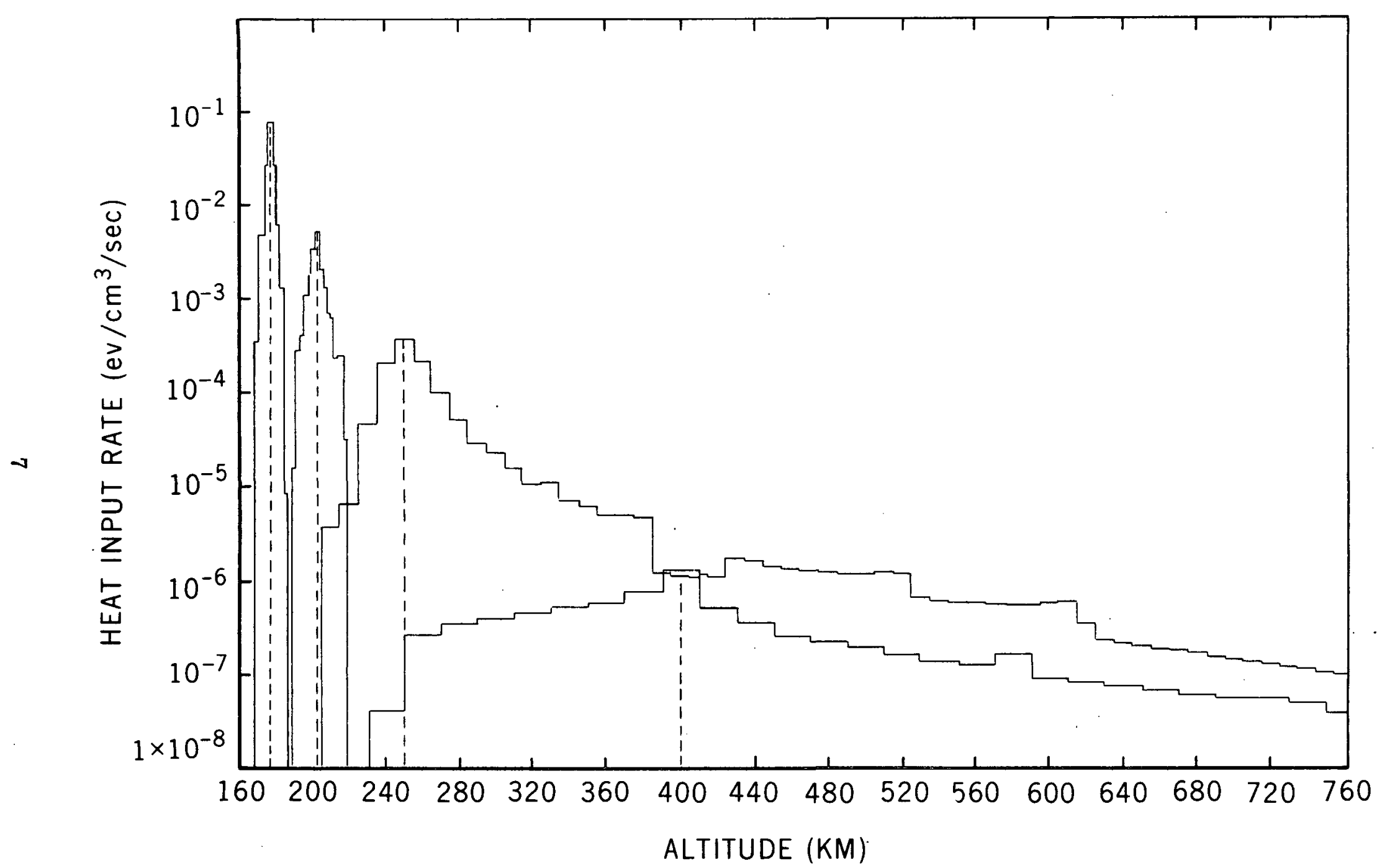

Figure 2. Heat input histograms generated by photoelectron production at four discrete altitudes. 


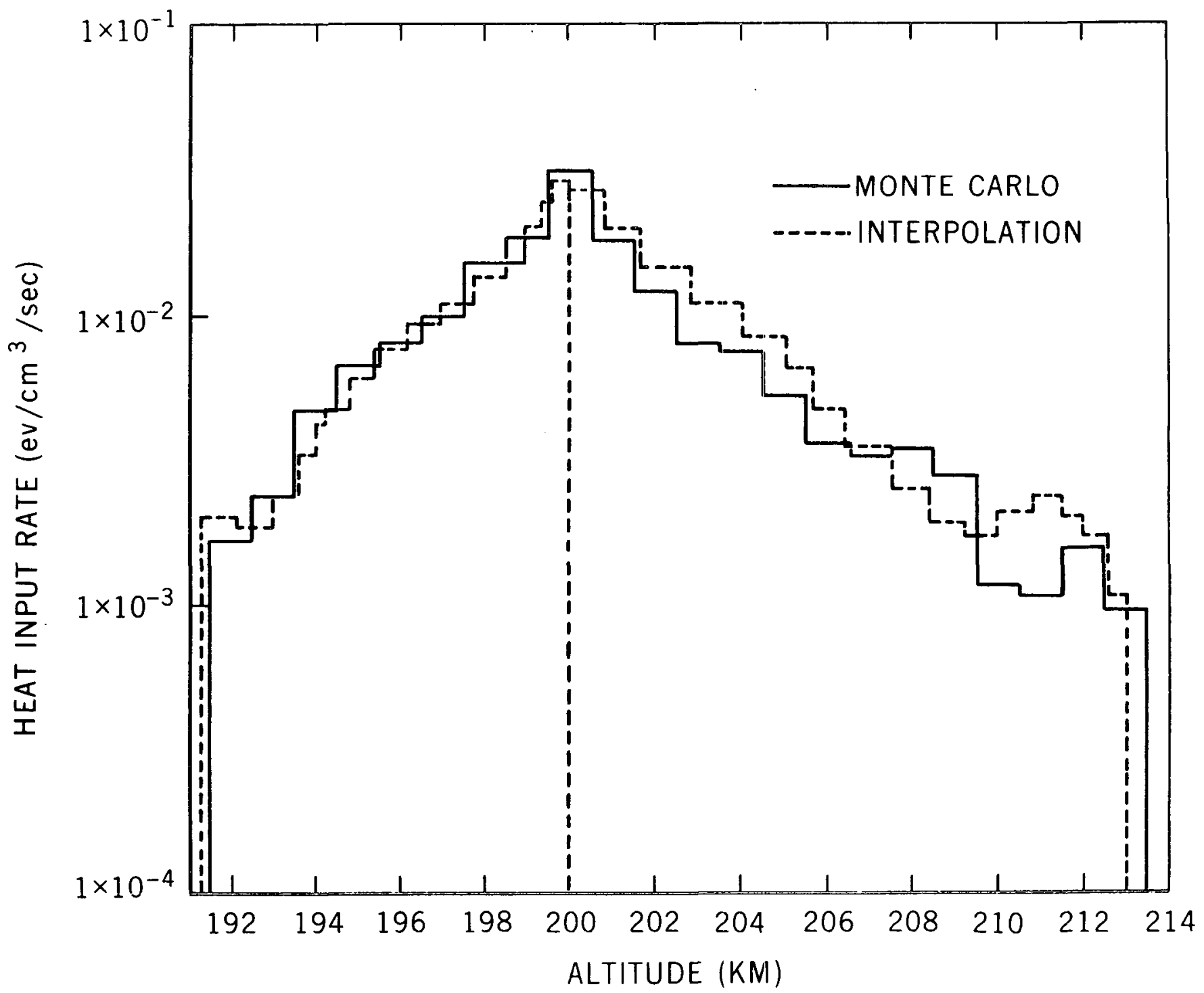

Figure 3. Comparison of a Monte Carlo generated heat input histogram with one obtained by interpolation. 


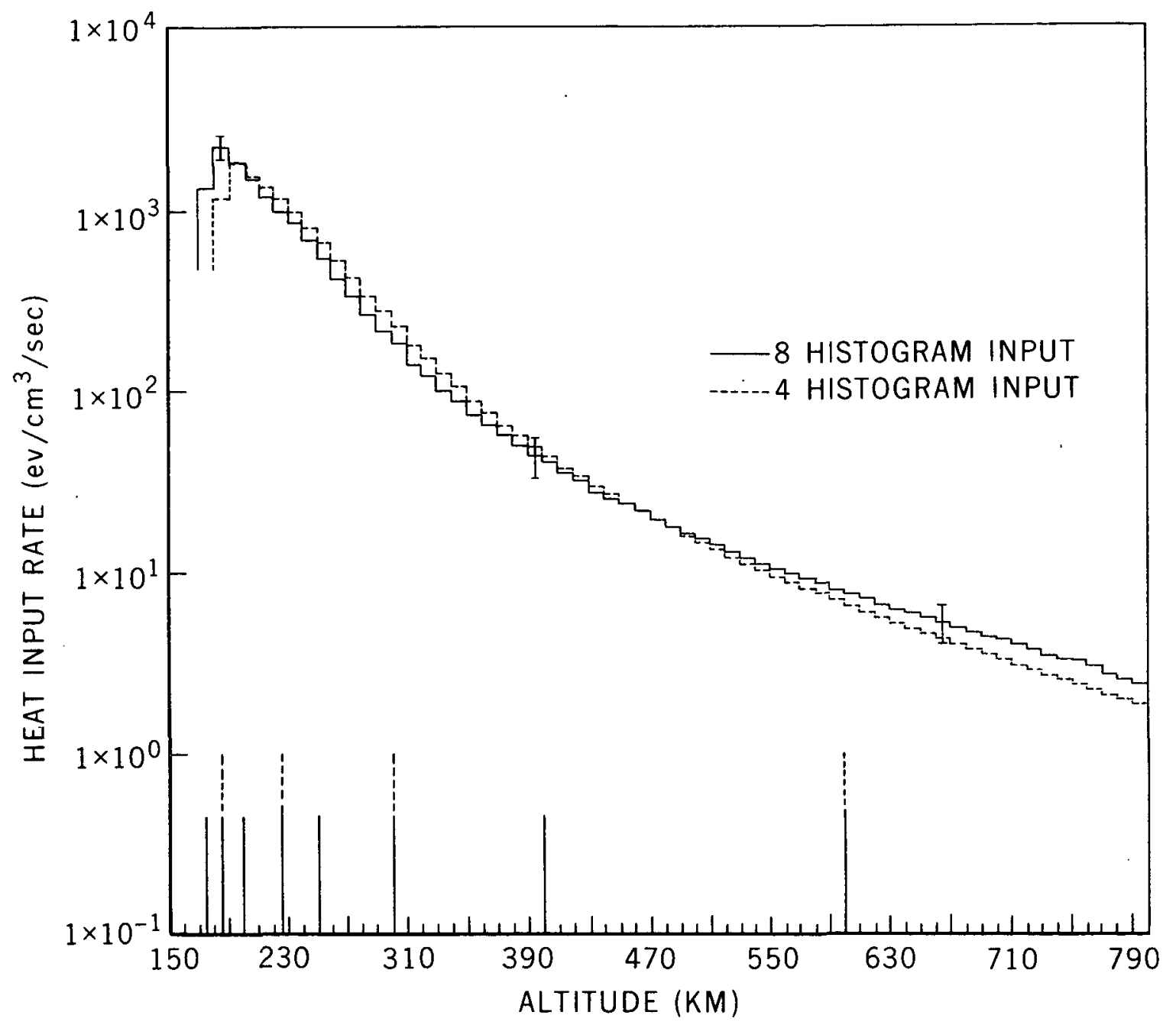

Figure 4. Comparison of a heat input profile based on 8 Monte Carlo histograms with one based on 4 Monte Carlo histograms. The solid (dashed) lines attached to the altitude axis indicate the altitudes at which photoelectrons are released in the case of 8 (4) Monte Carlo histograms. 